PROCEEDINGS OF THE

AMERICAN MATHEMATICAL SOCIETY

Volume 135, Number 7, July 2007, Pages 2165-2175

S 0002-9939(07)08811-9

Article electronically published on February 8, 2007

\title{
DRIFTED LAPLACE OPERATORS ON HOMOGENEOUS TREES
}

\author{
ENRICO CASADIO TARABUSI AND ALESSANDRO FIGÀ-TALAMANCA \\ (Communicated by Michael T. Lacey)
}

\begin{abstract}
We determine the spectrum and the resolvent operator of a drifted Laplace operator on a homogeneous tree, obtaining qualitatively different results according to the sign of the drift in the direction of a boundary point.
\end{abstract}

\section{INTRODUCTION}

Let $\mathfrak{T}$ be a homogeneous tree of degree $q+1$, and $\mathfrak{X}$ the set of its vertices. The (isotropic) Laplace operator on $\mathfrak{T}$ maps a complex-valued function $f$ on $\mathfrak{X}$ to the function $L f$ given by

$$
L f(x)=\frac{1}{q+1} \sum_{y \sim x} f(y) \quad \text { for every } x \in \mathfrak{X},
$$

where $y \sim x$ means that $y \in \mathfrak{X}$ is adjacent to $x$. In apparently different contexts, including its realization as a convolution operator on free groups, the operator $L$ (or $L-I$, as it is sometimes introduced) has been the object of thorough investigation, for instance in $[\mathrm{K}, \mathrm{C}, \mathrm{FP1}, \mathrm{MZ}, \mathrm{FP} 2, \mathrm{FN}$. A more complete list of references may be found in FP2, FS, FN.

A basic property of $L$ is its invariance with respect to the group Aut(T) of all automorphisms of the tree $\mathfrak{T}$. From this point of view it is natural to extend the investigation to average operators that are invariant for an appropriate subgroup $G$ of $\operatorname{Aut}(\mathfrak{T})$. This was done in $[\mathrm{A}, \mathrm{FS}$, for $G$ discrete, acting transitively on $\mathfrak{X}$, and isomorphic to a free group or a free power of the two-element group.

In this paper we study a class of drifted Laplace operators invariant for the group $G$ of automorphisms that fix a given element $\omega_{0}$ of the boundary $\Omega$ of $\mathfrak{T}$. Besides the choice of $\omega_{0}$, every such operator $L_{\theta}$ depends on a real parameter $\theta \in(0,1)$ that determines the $G$-invariant drift in the direction of $\omega_{0}$. Indeed, denoting by $x^{-}$the unique neighbor vertex of $x \in \mathfrak{X}$ that lies between $x$ and $\omega_{0}$, define

$$
L_{\theta} f(x)=\theta f\left(x^{-}\right)+\frac{1-\theta}{q} \sum_{y \in \mathfrak{X}: y^{-}=x} f(y) \quad \text { for every } x \in \mathfrak{X} .
$$

To a drifted Laplace operator is associated a nearest-neighbor random walk, once we interpret $\theta$ as the probability of moving from $x$ to $x^{-}$, and $(1-\theta) / q$ as the probability of moving from $x$ to each one of the other neighbors of $x$. The isotropic,

Received by the editors March 21, 2006.

2000 Mathematics Subject Classification. Primary 43A85; Secondary 05C05.

Key words and phrases. Homogeneous trees, Laplace operator, drifts, spectrum, resolvent operator. 
driftless case is the operator $L=L_{1 /(q+1)}$. In the case $\theta<1 /(q+1)$ the drift drags away from $\omega_{0}$, while in the case $\theta>1 /(q+1)$ the drift drags towards $\omega_{0}$. (According to some authors, the drift is absent for $\theta=1 / 2$ rather than for $\theta=1 /(q+1)$.)

For several questions in harmonic analysis a homogeneous tree can be considered as a natural discrete analogue of the hyperbolic half-plane $\Pi=\{x+i y \in \mathbf{C}: y>0\}$. The role of $\omega_{0}$ is played by the point at infinity, and that of $L_{\theta}$ by the drifted Laplace-Beltrami operator

$$
L_{b}=\frac{y^{2}}{2} \Delta+b y \frac{\partial}{\partial y}
$$

for real $b<1 / 2$. This operator is invariant for the group of isometries of $\Pi$ that fix $\infty$, i.e., the group $\{z \mapsto \alpha z+\beta, z \mapsto-\alpha \bar{z}+\beta: \alpha>0, \beta \in \mathbf{R}\}$. The analogy between the two settings was exploited in $\mathrm{BCF}$ (for $\theta<1 /(q+1)$ and $b<0$ ), where these drifted Laplace operators were used for parallel characterizations of symmetric stable distributions.

The main result of this paper (Theorem 3.2) is the computation of the spectrum of $L_{\theta}$ for every $0<\theta<1$ and of the resolvent operator, as well as, more generally, of the one-sided left or right resolvents where they exist (Proposition 3.4). These computations are carried out in several steps.

First of all we introduce, for any two vertices $x, y$ of the tree, a bi-distance $\mathbf{d}(x, y)$ as a pair of non-negative integers that express the relative position of $x, y$ with respect to $\omega_{0}$. The group $G$ is characterized as the group of all automorphisms of the tree that preserve $\mathbf{d}$. It becomes natural at this point to consider, for every pair $(j, k)$ of non-negative integers, the elementary summation operator $\Lambda_{j, k}$ whose kernel is the indicator function of the set $\{(x, y) \in \mathfrak{X} \times \mathfrak{X}: \mathbf{d}(x, y)=(j, k)\}$. The operators $\Lambda_{j, k}$ span the space of all $G$-invariant operators, and their norm is easily computed by using a result of L. Saloff-Coste and W. Woess [SW] concerning $G$-invariant operators with non-negative kernels. From this we finally prove, for every applicable $\gamma \in \mathbf{C}$, the boundedness of the natural candidates for the (two- or one-sided) inverse of the operator $\gamma-L_{\theta}$, which is of course $G$-invariant.

\section{Preliminaries}

It will be assumed that $q>1$, so that $\mathfrak{T}$ is infinite and not isomorphic to $\mathbf{Z}$. Unless otherwise stated, the notation and terminology of [FN] will be used. The choice of $\omega_{0}$ determines a partial ordering on $\mathfrak{X}$, by which $x \in \mathfrak{X}$ is an ancestor of $y \in \mathfrak{X}$ if $x$ lies on the half-geodesic $\left[y, \omega_{0}\right)$, the one-sided-infinite chain of vertices from $x$ to $\omega_{0}$. (The boundary point $\omega_{0}$ is sometimes called the mythical ancestor of all of $\mathfrak{X}$; cf. [C.) Each $x \in \mathfrak{X}$ can be identified with a subset of $\Omega^{\prime}=\Omega \backslash\left\{\omega_{0}\right\}$ : in this identification, a boundary point $\omega \neq \omega_{0}$ belongs to $x$ if the (doubly-infinite) geodesic $\left(\omega_{0}, \omega\right)$ from $\omega_{0}$ to $\omega$ contains $x$. Then $y \subseteq x$ if and only if $x$ is an ancestor of $y$, so the order relation is inclusion. The parent $x^{-}$of $x \in \mathfrak{X}$ is consequently the smallest vertex containing $x$ properly; of course $x$ is a child of $x^{-}$, and every vertex has exactly $q$ children. The union of two vertices is not always a vertex. In general, the join of $x, y \in \mathfrak{X}$ with respect to $\omega_{0}$ is the smallest vertex $x \vee y$ that contains $x, y$; similarly $x \vee \omega$ (respectively $\omega \vee \omega^{\prime}$ ) is the smallest vertex that contains $x \in \mathfrak{X}$ and $\omega \in \Omega^{\prime}$ (respectively $\omega, \omega^{\prime} \in \Omega^{\prime}$ ).

Let $\mathbf{N}$ be the set of non-negative integers. Let $d(x, y)$ be the usual tree distance between $x, y \in \mathfrak{X}$. We introduce their bi-distance (with respect to $\left.\omega_{0}\right) \mathbf{d}(x, y)=$ 


$$
\begin{aligned}
\left(d^{-}(x, y), d^{+}(x, y)\right) \in \mathbf{N} \times \mathbf{N} \text { by } & \\
& d^{-}(x, y)=d(x, x \vee y), \\
& d^{+}(x, y)=d(x \vee y, y)=d^{-}(y, x) .
\end{aligned}
$$

Consequently if $\mathbf{d}(x, y)=(j, k)$, then $\mathbf{d}(y, x)=(k, j)$. Furthermore

$$
d(x, y)=d^{-}(x, y)+d^{+}(x, y),
$$

because $x \vee y$ lies on the finite chain between $x, y$. An automorphism $g$ of $\mathfrak{T}$ belongs to $G$ if and only if it preserves bi-distances, i.e., $\mathbf{d}(g x, g y)=\mathbf{d}(x, y)$ for every $x, y \in \mathfrak{X}$. On the other hand, for every two pairs $(x, y),\left(x^{\prime}, y^{\prime}\right)$ of vertices such that $\mathbf{d}(x, y)=\mathbf{d}\left(x^{\prime}, y^{\prime}\right)$ there is $g \in G$ such that $g x=x^{\prime}$ and $g y=y^{\prime}$; that is, $\mathfrak{X}$ is two-point homogenous under the action of $G$ with respect to the bi-distance.

The relative horocycle index of $x, y \in \mathfrak{X}$ with respect to $\omega_{0}$ is

$$
h(x, y)=d^{-}(x, y)-d^{+}(x, y)=-h(y, x),
$$

and is readily proved to satisfy the cocycle relation $h(x, z)=h(x, y)+h(y, z)$ for every $x, y, z \in \mathfrak{X}$, so that $h(x, y)=0$ is an equivalence relation on $\mathfrak{X}$. The equivalence classes are the horocycles through $\omega_{0}$, and, once a base horocycle $\mathfrak{H}_{0}$ is arbitrarily chosen, each one may be indexed as $\mathfrak{H}_{n}$ with $n \in \mathbf{Z}$, adopting the convention that $n$ increases as the horocycle is farther from $\omega_{0}$; thus $h(x, y)=m-n$ for $x \in \mathfrak{H}_{m}$ and $y \in \mathfrak{H}_{n}$; cf. [FN, p. 35]. With respect to this choice of $\mathfrak{H}_{0}$ one can also define the absolute horocycle index of $x \in \mathfrak{X}$ with respect to $\omega_{0}$ by setting $h(x)=h(x, y)$ for any $y \in \mathfrak{H}_{0}$; i.e., $h(x)=n$ if $x \in \mathfrak{H}_{n}$.

The number of vertices that lie at bi-distance $(j, k)$ from a given vertex $x$, that is the cardinality $n_{j, k}$ of the set $\{y \in \mathfrak{X}: \mathbf{d}(x, y)=(j, k)\}$, is easily seen to be

$$
n_{j, k}= \begin{cases}q^{k} & \text { if } j=0 \text { or } k=0, \\ (q-1) q^{k-1} & \text { if } j, k>0 .\end{cases}
$$

A bounded linear operator $A$ on the space $\ell^{2}(\mathfrak{X})$ is associated to a summation kernel $A(x, y)=A \delta_{y}(x)$, where $\delta_{y}$ is the Kronecker delta at $y \in \mathfrak{X}$. If $f \in \ell^{2}(\mathfrak{X})$, then

$$
A f(x)=\sum_{y \in \mathfrak{X}} A(x, y) f(y) \quad \text { for every } x \in \mathfrak{X} .
$$

Therefore we can refer to $A$ as an operator or as a kernel. The adjoint $A^{*}$ of $A$ is associated to the kernel $A^{*}(x, y)=\overline{A(y, x)}$. The operator $A$ is invariant for $g \in \operatorname{Aut}(\mathfrak{X})$ if and only if its kernel is invariant under the diagonal action of $g$, that is, $A(g x, g y)=A(x, y)$ for every $x, y \in \mathfrak{X}$. A $G$-invariant operator $A$ has finite support if its kernel $A(x, y)$ vanishes for large enough $d(x, y)$.

We shall make use of the elementary $G$-invariant kernels that take the value 1 on pairs of vertices at a given bi-distance, and vanish otherwise. For $j, k \in \mathbf{N}$ let $\Lambda_{j, k}$ be the $G$-invariant operator given for $x, y \in \mathfrak{X}$ by

$$
\Lambda_{j, k}(x, y)= \begin{cases}1 & \text { if } \mathbf{d}(x, y)=(j, k), \\ 0 & \text { if } \mathbf{d}(x, y) \neq(j, k) .\end{cases}
$$


Lemma 2.1. The operator norm of $\Lambda_{j, k}$ on $\ell^{2}(\mathfrak{X})$ is

$$
\left\|\Lambda_{j, k}\right\|=\sqrt{n_{j, k} n_{k, j}}=q^{(j+k) / 2} \cdot \begin{cases}1 & \text { if } j=0 \text { or } k=0, \\ \frac{q-1}{q} & \text { if } j, k>0 .\end{cases}
$$

Moreover

$$
\Lambda_{j, k}^{*}=\Lambda_{k, j} \quad \text { for every } j, k \in \mathbf{N},
$$

and

$$
\Lambda_{j, k}= \begin{cases}\text { identity } & \text { if } j=k=0, \\ \Lambda_{1,0}^{j} & \text { if } k=0, \\ \Lambda_{0,1}^{k} & \text { if } j=0 \\ \Lambda_{1,0}^{j} \Lambda_{0,1}^{k}-\Lambda_{1,0}^{j-1} \Lambda_{0,1}^{k-1} & \text { if } j, k>0 .\end{cases}
$$

We also have

$$
\begin{aligned}
& \Lambda_{j, k} \Lambda_{1,0}= \begin{cases}\Lambda_{j+1,0} & \text { if } k=0, \\
(q-1) \Lambda_{j, 0} & \text { if } k=1 \text { and } j>0, \\
q \Lambda_{j, k-1} & \text { if } k>j=0 \text { or } k>1,\end{cases} \\
& \Lambda_{j, k} \Lambda_{0,1}= \begin{cases}\Lambda_{j-1,0}+\Lambda_{j, 1} & \text { if } j>0=k, \\
\Lambda_{j, k+1} & \text { if } j=0 \text { or } k>0 ;\end{cases}
\end{aligned}
$$

similar expressions for $\Lambda_{1,0} \Lambda_{j, k}$ and $\Lambda_{0,1} \Lambda_{j, k}$ may be obtained from (2.5) by using (2.3). In particular

$$
\begin{aligned}
& \Lambda_{1,0} \Lambda_{0,1}=\Lambda_{0,0}+\Lambda_{1,1}, \\
& \Lambda_{0,1} \Lambda_{1,0}=q \Lambda_{0,0},
\end{aligned}
$$

whence the commutator of $\Lambda_{1,0}, \Lambda_{0,1}$ is

$$
\left[\Lambda_{1,0}, \Lambda_{0,1}\right]=\Lambda_{1,1}-(q-1) \Lambda_{0,0} .
$$

Proof. We shall prove (2.2) only; the remaining identities follow from direct computation. Fix a Haar measure $|\cdot|$ on $G$, and let $G_{x}$ be the isotropy subgroup of $G$ at $x \in \mathfrak{X}$. Then $\left|G_{y}\right| /\left|G_{x}\right|=q^{h(x, y)}$ for every $y \in \mathfrak{X}$. By $[\mathrm{SW}$, (3.3) of Theorem 3.1], for any $x \in \mathfrak{X}$ we have

$$
\left\|\Lambda_{j, k}\right\|=\sum_{y \in \mathfrak{X}} \sqrt{\frac{\left|G_{y}\right|}{\left|G_{x}\right|}} \Lambda_{j, k}(x, y)=\sum_{\substack{y \in \mathfrak{X}: \\ \mathbf{d}(x, y)=(j, k)}} \sqrt{q^{j-k}}=n_{j, k} q^{(j-k) / 2},
$$

which by (2.1) yields (2.2).

The value $A(x, y)$ of the kernel of a $G$-invariant bounded operator $A$ on $\ell^{2}(\mathfrak{X})$ only depends on $\mathbf{d}(x, y)$, so that for each $j, k \in \mathbf{N}$ we can set

$$
a_{j, k}=A(x, y) \quad \text { for any } x, y \in \mathfrak{X} \text { such that } \mathbf{d}(x, y)=(j, k) .
$$

Thus we can write

$$
A(x, y)=\sum_{j, k=0}^{\infty} a_{j, k} \Lambda_{j, k}(x, y) \quad \text { for every } x, y \in \mathfrak{X}
$$


and the operator $A$ is identified with the infinite matrix $\left(a_{j, k}\right)_{j, k \in \mathbf{N}}$. If $a_{j, k} \geq 0$ for every $j, k \in \mathbf{N}$, again [SW, (3.3) of Theorem 3.1] implies that

$$
\|A\|=\sum_{j, k=0}^{\infty} n_{j, k} q^{(j-k) / 2} a_{j, k} \leq \sum_{j, k=0}^{\infty} a_{j, k} q^{(j+k) / 2} .
$$

More generally, in view of (2.2) we derive the following result.

Corollary 2.2. Assume given $a_{j, k} \in \mathbf{C}$ for every $j, k \in \mathbf{N}$, and suppose that

$$
\sum_{j, k=0}^{\infty}\left|a_{j, k}\right| q^{(j+k) / 2}<\infty
$$

Then the series

$$
A=\sum_{j, k=0}^{\infty} a_{j, k} \Lambda_{j, k}
$$

is convergent in the $\ell^{2}(\mathfrak{X})$ operator norm to a bounded $G$-invariant operator $A$ with

$$
\|A\| \leq \sum_{j, k=0}^{\infty}\left|a_{j, k}\right| q^{(j+k) / 2}
$$

The kernel of the drifted Laplace operator $L_{\theta}$ under investigation in this paper is given by

$$
L_{\theta}(x, y)= \begin{cases}\theta & \text { if } y=x^{-} \\ (1-\theta) / q & \text { if } x=y^{-} \\ 0 & \text { otherwise }\end{cases}
$$

We first show that $L_{\theta}$ itself and its adjoint $L_{\theta}^{*}$ generate (as do the elementary operators $\left.\Lambda_{1,0}, \Lambda_{0,1}\right)$ all $G$-invariant operators.

Proposition 2.3. For $\theta \neq 1 /(q+1)$ the algebra of polynomials in $L_{\theta}, L_{\theta}^{*}$ is weakly dense in the von Neumann algebra of $G$-invariant operators on $\ell^{2}(\mathfrak{X})$.

Proof. Clearly $L_{\theta}, L_{\theta}^{*}$ may be expressed as linear combinations of the elementary operators $\Lambda_{1,0}, \Lambda_{1,0}$ as

$$
\begin{aligned}
& L_{\theta}=\theta \Lambda_{1,0}+\frac{1-\theta}{q} \Lambda_{0,1}, \\
& L_{\theta}^{*}=\frac{1-\theta}{q} \Lambda_{1,0}+\theta \Lambda_{0,1} .
\end{aligned}
$$

It follows that, if $\theta \neq 1 /(q+1)$, then $L_{\theta}, L_{\theta}^{*}$ are linearly independent, so that $\Lambda_{1,0}, \Lambda_{0,1}$ may conversely be expressed as linear combinations of $L_{\theta}, L_{\theta}^{*}$.

Every finitely supported $G$-invariant operator is a linear combination of the operators $\Lambda_{j, k}$ for $j, k \in \mathbf{N}$, which are expressed as polynomials in $\Lambda_{1,0}, \Lambda_{0,1}$ by (2.4). Therefore every $G$-invariant operator of finite support is a polynomial in $L_{\theta}, L_{\theta}^{*}$.

It remains to prove that every bounded $G$-invariant operator on $\ell^{2}(\mathfrak{X})$ may be approximated in the weak topology by finitely supported $G$-invariant operators. Indeed $G$-invariant operators may be put into a one-to-one correspondence with right convolution operators on $L^{2}(G)$ with respect to the left Haar measure [SW]. This correspondence is isometric and maps finitely supported operators onto the algebra of right convolution operators by continuous functions on $G$ with compact 
support, which is dense in the von Neumann algebra of operators on $L^{2}(G)$ that commute with left translations.

Remark 2.4. As shown in the proof above, the algebra of polynomials in $L_{\theta}, L_{\theta}^{*}$ coincides with the algebra of polynomials in $\Lambda_{1,0}, \Lambda_{0,1}$. From (2.6) the relation between commutators is

$$
\left[L_{\theta}, L_{\theta}^{*}\right]=\left(\theta^{2}-\frac{(1-\theta)^{2}}{q^{2}}\right)\left[\Lambda_{1,0}, \Lambda_{0,1}\right] .
$$

The following remark shows that the adjoint $L_{\theta}^{*}$ of $L_{\theta}$ is a multiple of $L_{\theta^{\prime}}$ for a suitable drift $\theta^{\prime}$, with $\theta^{\prime} \neq \theta$ unless $\theta=1 /(q+1)$.

Remark 2.5. For $0<\theta<1$, if

$$
b_{\theta}=q \theta+\frac{1-\theta}{q}, \quad \theta^{\prime}=\frac{1}{1+q^{2} \theta /(1-\theta)},
$$

then

$$
L_{\theta}^{*}=b_{\theta} L_{\theta^{\prime}} .
$$

The function $\theta \mapsto \theta^{\prime}$ is strictly decreasing on the interval $(0,1)$ onto itself, so that $\theta, \theta^{\prime}$ always lie on opposite sides with respect to the fixed point $1 /(q+1)$.

For $a>0$ denote by $M_{a}$ the operator of multiplication by $a^{h}$; observe that $M_{a}^{-1}=M_{1 / a}$. The following result may be regarded as a direct consequence of the reversibility of the random walk associated to $L_{\theta}$; cf. [W, p. 126].

Proposition 2.6. For $0<\theta<1$, if

$$
a=\sqrt{\frac{1-\theta}{\theta q}}, \quad \mu=(q+1) \sqrt{\frac{\theta(1-\theta)}{q}},
$$

then

$$
L_{\theta}=\mu M_{a}^{-1} L M_{a} .
$$

Therefore a (not necessarily bounded) function $f$ is an eigenfunction of $L$ of eigenvalue $\lambda$ if and only if $M_{1 / a} f$ is an eigenfunction of $L_{\theta}$ of eigenvalue $\mu \lambda$.

Proof. For every $x \in \mathfrak{X}$ we have

$$
\begin{aligned}
\mu M_{a}^{-1} L M_{a} f(x) & =\mu a^{-h(x)} L\left(a^{h} f\right)(x) \\
& =\mu a^{-h(x)} \frac{1}{q+1}\left(a^{h\left(x^{-}\right)} f\left(x^{-}\right)+\sum_{y^{-}=x} a h(y) f(y)\right) \\
& =\frac{\mu}{q+1}\left(\frac{f\left(x^{-}\right)}{a}+\sum_{y^{-}=x} a f(y)\right) \\
& =\theta f\left(x^{-}\right)+\frac{1-\theta}{q} \sum_{y^{-}=x} f(y)=L_{\theta} f(x) .
\end{aligned}
$$




\section{The SPECTRUM AND THE RESOLVENT OPERATOR}

For $0<\theta<1$, consider the function $\phi$ given by

$$
\phi(\zeta)=(1-\theta) \zeta+\theta / \zeta \quad \text { for every } \zeta \in \mathbf{C}^{*}
$$

This is a two-to-one holomorphic mapping of $\mathbf{C}^{*}$ onto $\mathbf{C}$ with branching points at $\pm \sqrt{\theta /(1-\theta)}$. For every $\rho>0$ it maps the circle $C_{\rho}$ of radius $\rho$ and center at the origin - as well as the circle $C_{\theta /(1-\theta) \rho}$-onto the ellipse $\phi\left(C_{\rho}\right)=\phi\left(C_{\theta /(1-\theta) \rho}\right)$ with foci at

$$
\begin{aligned}
& F_{+}=+2 \sqrt{\theta(1-\theta)}, \\
& F_{-}=-2 \sqrt{\theta(1-\theta)},
\end{aligned}
$$

both independent of $\rho$, and with shorter (i.e., vertical) semi-axis of length

$$
\sigma=|\phi(i \rho)|=|(1-\theta) \rho-\theta / \rho| \quad \text { for every } \rho>0
$$

denote by $\mathcal{E}$ the family of these confocal ellipses. Conversely, the dependence of the two values $\rho_{1}, \rho_{2}$ of $\rho$ on $\sigma$, with $\rho_{1} \leq \rho_{2}$, is given by

$$
\begin{array}{ll}
\rho_{1}(\sigma)=\frac{-\sigma+\sqrt{\sigma^{2}+4 \theta(1-\theta)}}{2(1-\theta)} & \text { for every } \sigma \geq 0, \\
\rho_{2}(\sigma)=\frac{+\sigma+\sqrt{\sigma^{2}+4 \theta(1-\theta)}}{2(1-\theta)} & \text { for every } \sigma \geq 0 .
\end{array}
$$

The function $\rho_{1}$ is strictly decreasing and the function $\rho_{2}$ strictly increasing on $[0,+\infty)$. They agree at $\sigma=0$, because the circle of radius $\rho_{ \pm}(0)=\sqrt{\theta /(1-\theta)}$ is mapped onto the degenerate ellipse equal to the segment joining $F_{+}, F_{-}$.

Let us introduce the constant

$$
c_{\theta}=\frac{\theta q}{1-\theta} \begin{cases}<1 & \text { if } \theta<1 /(q+1) \\ =1 & \text { if } \theta=1 /(q+1) \\ >1 & \text { if } \theta>1 /(q+1)\end{cases}
$$

and single out the ellipse

$$
E=\phi\left(C_{1 / \sqrt{q}}\right)=\phi\left(C_{c_{\theta} / \sqrt{q}}\right)
$$

Let $E^{\text {int }}, E^{\text {ext }}$ be the bounded, respectively unbounded component of the complement of $E$, and let

$$
\hat{\sigma}=|\theta \sqrt{q}-(1-\theta) / \sqrt{q}|
$$

be the value of $\sigma$ on $E$. If $\theta=1 /(q+1)$, then $\hat{\sigma}=0$ and the ellipse $E$ degenerates to the segment, thus $E^{\text {int }}$ is empty. 
Lemma 3.1. For $\gamma \in \mathbf{C}$ let $\zeta_{1}, \zeta_{2}$ be the solutions of the quadratic equation $\phi(\zeta)=$ $\gamma$, with $\left|\zeta_{1}\right| \leq\left|\zeta_{2}\right|$, and set $\eta_{j}=\zeta_{j} / c_{\theta}$ for $j=1,2$. We have

$$
\begin{array}{ll}
\left|\zeta_{1}\right|<\left|\eta_{1}\right|<1 / \sqrt{q}<\left|\zeta_{2}\right|<\left|\eta_{2}\right| & \text { if } \gamma \in E^{\text {ext }} \quad \text { and } \theta<1 /(q+1), \\
\left|\zeta_{1}\right|=\left|\eta_{1}\right|<1 / \sqrt{q}<\left|\zeta_{2}\right|=\left|\eta_{2}\right| & \text { if } \gamma \in E^{\text {ext }} \text { and } \theta=1 /(q+1), \\
\left|\eta_{1}\right|<\left|\zeta_{1}\right|<1 / \sqrt{q}<\left|\eta_{2}\right|<\left|\zeta_{2}\right| & \text { if } \gamma \in E^{\text {ext }} \text { and } \theta>1 /(q+1), \\
\left|\zeta_{1}\right|<\left|\zeta_{2}\right|=1 / \sqrt{q}=\left|\eta_{1}\right|<\left|\eta_{2}\right| & \text { if } \gamma \in E \text { and } \theta<1 /(q+1), \\
\left|\zeta_{1}\right|=\left|\zeta_{2}\right|=1 / \sqrt{q}=\left|\eta_{1}\right|=\left|\eta_{2}\right| & \text { if } \gamma \in E \text { and } \theta=1 /(q+1), \\
\left|\eta_{1}\right|<\left|\eta_{2}\right|=1 / \sqrt{q}=\left|\zeta_{1}\right|<\left|\zeta_{2}\right| & \text { if } \gamma \in E \text { and } \theta>1 /(q+1), \\
\left|\zeta_{1}\right| \leq\left|\zeta_{2}\right|<1 / \sqrt{q}<\left|\eta_{1}\right| \leq\left|\eta_{2}\right| & \text { if } \gamma \in E^{\text {int }} \text { and } \theta<1 /(q+1), \\
\left|\eta_{1}\right| \leq\left|\eta_{2}\right|<1 / \sqrt{q}<\left|\zeta_{1}\right| \leq\left|\zeta_{2}\right| & \text { if } \gamma \in E^{\text {int }} \text { and } \theta>1 /(q+1) .
\end{array}
$$

Proof. Because $\phi$ maps circles to ellipses, the values of $\left|\zeta_{1}\right|,\left|\zeta_{2}\right|,\left|\eta_{1}\right|,\left|\eta_{2}\right|$ only depend on which ellipse of the family $\mathcal{E}$ contains $\gamma$, and such an ellipse is uniquely identified by the length $\sigma$ of its shorter semi-axis. Indeed $\left|\zeta_{j}\right|=\rho_{j}(\sigma)$ and $\left|\eta_{j}\right|=\rho_{j}(\sigma) / c_{\theta}$ for $j=1,2$.

If $\gamma \in E$, then $\sigma=\hat{\sigma}$, therefore $\left\{\rho_{1}(\sigma), \rho_{2}(\sigma)\right\}=\left\{1 / \sqrt{q}, c_{\theta} / \sqrt{q}\right\}$, and (3.1) gives the required inequalities for this case.

If $\gamma \in E^{\text {ext }}$, then $\sigma>\hat{\sigma}$, therefore $\rho_{1}(\sigma)<\rho_{1}(\hat{\sigma}) \leq 1 / \sqrt{q} \leq \rho_{2}(\hat{\sigma})<\rho_{2}(\sigma)$, and similarly $\rho_{1}(\sigma) / c_{\theta}<\rho_{1}(\hat{\sigma}) / c_{\theta} \leq 1 / \sqrt{q} \leq \rho_{2}(\hat{\sigma}) / c_{\theta}<\rho_{2}(\sigma) / c_{\theta}$, which, together with (3.1), completes the proof for this case.

Finally, if $\gamma \in E^{\text {int }}$, then $\sigma<\hat{\sigma}$. If, in addition, $\theta<1 /(q+1)$, then $\rho_{2}(\sigma)<$ $\rho_{2}(\hat{\sigma})=1 / \sqrt{q}=\rho_{1}(\hat{\sigma}) / c_{\theta}<\rho_{1}(\sigma) / c_{\theta}$, again by (3.1). On the other hand, if $\theta>1 /(q+1)$ we conclude in a similar fashion.

Theorem 3.2. For $0<\theta<1$, the spectrum of the operator $L_{\theta}$ is the filled ellipse $E \cup E^{\mathrm{int}}$. For $\gamma$ in its complement $E^{\mathrm{ext}}$, the resolvent $R=\left(\gamma-L_{\theta}\right)^{-1}$ is the $G$-invariant operator defined by the kernel

$$
R(x, y)=\sum_{j, k=0}^{\infty} r_{j, k} \Lambda_{j, k}(x, y) \quad \text { for every } x, y \in \mathfrak{X}
$$

with

$$
r_{j, k}=\beta \zeta_{1}^{j} \eta_{1}^{k} \quad \text { for every }(j, k) \in \mathbf{N} \times \mathbf{N},
$$

where $\zeta_{1}$ is the root of smallest modulus of the quadratic equation $\phi(\zeta)=\gamma$, where $\eta_{1}=\zeta_{1} / c_{\theta}$, and where the normalization constant $\beta$ is given by

$$
\gamma=\left(1+\frac{1}{q}\right)(1-\theta) \zeta_{1}+\frac{1}{\beta}
$$

This includes the case $\theta=1 /(q+1)$, where $\zeta_{1}=\eta_{1}$ and the spectrum reduces to the real segment between the points $F_{ \pm}= \pm 2 \sqrt{q} /(q+1) ;$ cf. [K].

Proof. The kernel $R(x, y)$ represents the required resolvent for some $\gamma \in \mathbf{C}$ if and only if it satisfies the resolvent relations $R\left(\gamma-L_{\theta}\right)=\Lambda_{0,0}=\left(\gamma-L_{\theta}\right) R$ and defines a bounded operator on $\ell^{2}(\mathfrak{X})$. (These relations may in principle be satisfied by $G$-invariant kernels that do not represent bounded operators.) In view of relations (2.5) and expression (2.6) for the Laplace operator, the resolvent relations 
respectively expand to

$$
\begin{aligned}
& \gamma r_{j, k}= \begin{array}{ll}
\frac{1-\theta}{q} r_{1,0}+q \theta r_{0,1}+1 & \text { if } j=k=0, \\
\frac{1-\theta}{q} r_{j+1,0}+\theta r_{j-1,0}+(q-1) \theta r_{j, 1} & \text { if } j>k=0, \\
\frac{1-\theta}{q} r_{j, k-1}+q \theta r_{j, k+1} & \text { if } k>0,
\end{array} \\
& \gamma r_{j, k}= \begin{cases}(1-\theta) r_{1,0}+\theta r_{0,1}+1 & \text { if } j=k=0, \\
\frac{q-1}{q}(1-\theta) r_{1, k}+\frac{1-\theta}{q} r_{0, k-1}+\theta r_{0, k+1} & \text { if } j=0<k, \\
(1-\theta) r_{j+1, k}+\theta r_{j-1, k} & \text { if } j>0 .\end{cases}
\end{aligned}
$$

By (3.3), if $r_{j, k}$ vanished for all $k>0$ and all $j$, then so would $r_{j, 0}$ for all $j$. Thus $r_{j, k} \neq 0$ for some $j$ and some positive $k$. For fixed $j$, the general solution of the difference equation (3.3) in the variable $k$ is

$$
r_{j, k}= \begin{cases}c_{j} \eta_{1}^{k}+c_{j}^{\prime} \eta_{2}^{k} & \text { if } \eta_{1}, \eta_{2} \text { are distinct roots of } \phi\left(c_{\theta} \eta\right)=\gamma, \\ c_{j} \eta^{k}+c_{j}^{\prime} k \eta^{k} & \text { if } \eta \text { is a double root of } \phi\left(c_{\theta} \eta\right)=\gamma,\end{cases}
$$

where $c_{j}, c_{j}^{\prime}$ are constants depending on $j$. Likewise the general solution of the difference equation (3.4) in the variable $j$ is

$$
r_{j, k}= \begin{cases}d_{k} \zeta_{1}^{j}+d_{k}^{\prime} \zeta_{2}^{j} & \text { if } \zeta_{1}, \zeta_{2} \text { are distinct roots of } \phi(\zeta)=\gamma, \\ d_{k} \zeta^{j}+d_{k}^{\prime} j \zeta^{j} & \text { if } \zeta \text { is a double root of } \phi(\zeta)=\gamma,\end{cases}
$$

with $d_{k}, d_{k}^{\prime}$ constants.

If $\gamma \in E^{\text {ext }}$, each of the quadratic equations $\phi(\zeta)=\gamma$ and $\phi\left(c_{\theta} \eta\right)=\gamma$ has distinct roots, because double roots occur only at $\gamma=F_{ \pm}$, the foci of $E$. The kernel given by (3.2) satisfies (3.5), (3.6). To prove that it defines a bounded operator, in view of Corollary 2.2 it suffices to show that $\left|\zeta_{1}\right|,\left|\eta_{1}\right|<1 / \sqrt{q}$. This is done in Lemma3.1.

Assume instead that $\gamma \in E \cup E^{\text {int }}$. If $R$ is an inverse of $\gamma-L_{\theta}$, then its coefficients $r_{j, k}$ must be expressed as in (3.5), (3.6). On the other hand, $R$ is bounded, which implies that $R \delta_{x}, R^{*} \delta_{x}$ belong to $\ell^{2}$. Since $R^{*} \delta_{x}(y)=\overline{R(x, y)}$, this yields

$$
\begin{array}{ll}
\sum_{j=0}^{\infty}\left|r_{j, k}\right|^{2} q^{j}<\infty & \text { for every } k \in \mathbf{N}, \\
\sum_{k=0}^{\infty}\left|r_{j, k}\right|^{2} q^{k}<\infty & \text { for every } j \in \mathbf{N} .
\end{array}
$$

By Lemma 3.1 if $\theta<1 /(q+1)$, then $\left|\eta_{1}\right|,\left|\eta_{2}\right| \geq 1 / \sqrt{q}$, and the latter series is divergent; while if $\theta>1 /(q+1)$, then $\left|\zeta_{1}\right|,\left|\zeta_{2}\right| \geq 1 / \sqrt{q}$, and the former series is divergent.

Notation 3.3. In the sequel, in order to avoid exceptions for double roots, we set

$$
\frac{\alpha_{1}^{j}-\alpha_{2}^{j}}{\alpha_{1}-\alpha_{2}}=j \alpha^{j-1} \quad \text { if } \alpha_{1}=\alpha_{2}=\alpha, \text { and } j \in \mathbf{N} .
$$


Proposition 3.4. Assume that $\gamma$ lies in the interior $E^{\text {int }}$ of the spectrum of $L_{\theta}$.

If $\theta<1 /(q+1)$, then $\gamma-L_{\theta}$ has a right inverse $R^{\mathfrak{r}}$ given by

$$
R^{\mathfrak{r}}(x, y)=\sum_{j, k=0}^{\infty} r_{j, k}^{\mathfrak{r}} \Lambda_{j . k}
$$

where (with Notation 3.3)

$$
r_{j, k}^{\mathfrak{r}}= \begin{cases}-\frac{1}{1-\theta} \frac{\zeta_{1}^{j}-\zeta_{2}^{j}}{\zeta_{1}-\zeta_{2}} & \text { if } j>k=0, \\ 0 & \text { if } j=0 \text { or } k>0,\end{cases}
$$

and where $\zeta_{1}, \zeta_{2}$ are the solutions of $\phi(\zeta)=\gamma$.

On the other hand, if $\theta>1 /(q+1)$, then $\gamma-L_{\theta}$ has a left inverse $R^{\mathfrak{l}}$ given by

$$
R^{\mathfrak{l}}(x, y)=\sum_{j, k=0}^{\infty} r_{j, k}^{\mathfrak{l}} \Lambda_{j, k}
$$

where (again with Notation 3.3)

$$
r_{j, k}^{\mathfrak{r}}= \begin{cases}-\frac{1}{\theta q} \frac{\eta_{1}^{k}-\eta_{2}^{k}}{\eta_{1}-\eta_{2}} & \text { if } k>j=0, \\ 0 & \text { if } k=0 \text { or } j>0,\end{cases}
$$

and where $\eta_{1}, \eta_{2}$ are the solutions of $\phi\left(c_{\theta} \eta\right)=\gamma$.

Proof. If $\theta>1 /(q+1)$, then $\left|\eta_{1}\right|,\left|\eta_{2}\right|<1 / \sqrt{q}$ by Lemma 3.1. Therefore $r^{\mathfrak{l}}$ satisfies (3.3) and

$$
\left|r_{0, k}^{\mathfrak{l}}\right| \leq \frac{1}{q \theta} k\left|\eta_{2}\right|^{k-1} \quad \text { for every } k>0 .
$$

By Corollary 2.2 we obtain

$$
\|R\| \leq \sum_{j, k=0}^{\infty}\left\|r_{j, k}^{\mathfrak{l}} \Lambda_{j, k}\right\| \leq \sum_{k=1}^{\infty}\left|r_{0, k}^{\mathfrak{l}}\right| q^{k / 2} \leq \frac{1}{\sqrt{q} \theta} \sum_{k=1}^{\infty} k\left(\sqrt{q}\left|\eta_{1}\right|\right)^{k-1}<\infty .
$$

We conclude that $R^{\mathfrak{l}}$ is a left inverse of $\gamma-L_{\theta}$.

If $\theta<1 /(q+1)$, then $\left|\zeta_{1}\right|,\left|\zeta_{2}\right|<1 / \sqrt{q}$ by Lemma 3.1. Therefore $r^{\mathfrak{r}}$ satisfies (3.4). By Corollary 2.2 it defines a bounded operator which is a right inverse for $\gamma-L_{\theta}$.

Remark 3.5. By Theorem 3.2 and Proposition 3.4, if $0<\theta<1 /(q+1)$ the interior $E^{\text {int }}$ of the spectrum of $L_{\theta}$ is contained in the point spectrum. If $x_{0}, x_{0}^{\prime} \in \mathfrak{X}$ are such that $\mathbf{d}\left(x_{0}, x_{0}^{\prime}\right)=(1,1)$ (i.e., they are distinct children of a same parent vertex) and if $\zeta_{1}, \zeta_{2}$ are the solutions of $\phi(\zeta)=\gamma$, an $\ell^{2}(\mathfrak{X})$ eigenfunction $f$ of eigenvalue $\gamma \in E^{\text {int }}$ is easily seen to be given by (always with Notation 3.3 )

$$
f(x)= \begin{cases}\frac{\zeta_{1}^{k+1}-\zeta_{2}^{k+1}}{\zeta_{1}-\zeta_{2}} & \text { if } \mathbf{d}\left(x_{0}, x\right)=(0, k), \\ -\frac{\zeta_{1}^{k+1}-\zeta_{2}^{k+1}}{\zeta_{1}-\zeta_{2}} & \text { if } \mathbf{d}\left(x_{0}^{\prime}, x\right)=(0, k), \\ 0 & \text { otherwise. }\end{cases}
$$


On the other hand, if $1 /(q+1)<\theta<1$, the interior of the spectrum is contained in the residual spectrum [RS, VI.3 p. 188]. Of course the spectrum has empty interior if $\theta=1 /(q+1)$.

\section{REFERENCES}

[A] K. Aomoto, Spectral theory on a free group and algebraic curves, J. Fac. Sci. Univ. Tokyo Sect. IA Math. 31 (1984), no. 2, 297-318. MR763424 (86m:58127)

[BCF] P. Baldi, E. Casadio Tarabusi, and A. Figà-Talamanca, Stable laws arising from hitting distributions of processes on homogeneous trees and the hyperbolic half-plane, Pacific J. Math. 197 (2001), no. 2, 257-273. MR1815256 (2001m:60015)

[C] P. Cartier, Harmonic analysis on trees, Harmonic analysis on homogeneous spaces (Williams Coll., Williamstown, Mass., 1972), Proc. Sympos. Pure Math., vol. 26, Amer. Math. Soc., Providence, 1973, pp. 419-424. MR0338272 (49:3038)

[FN] A. Figà-Talamanca and C. Nebbia, Harmonic analysis and representation theory for groups acting on homogeneous trees, London Math. Soc. Lecture Note Ser., vol. 162, Cambridge Univ. Press, Cambridge, 1991. MR.1152801 (93f:22004)

[FP1] A. Figà-Talamanca and M. A. Picardello, Spherical functions and harmonic analysis on free groups, J. Funct. Anal. 47 (1982), no. 3, 281-304. MR665019 (83m:22018)

[FP2] - Harmonic analysis on free groups, Lecture Notes in Pure and Appl. Math., vol. 87, Dekker, New York, 1983. MR710827 (85j:43001)

[FS] A. Figà-Talamanca and T. Steger, Harmonic analysis for anisotropic random walks on homogeneous trees, Mem. Amer. Math. Soc. 110 (1994), no. 531, xii+68. MR1219707 (95a:22003)

[K] H. Kesten, Symmetric random walks on groups, Trans. Amer. Math. Soc. 92 (1959), 336354. MR0109367(22:253)

[MZ] A. M. Mantero and A. Zappa, The Poisson transform and representations of a free group, J. Funct. Anal. 51 (1983), no. 3, 372-399. MR703084 (85b:22010)

[RS] M. C. Reed and B. Simon, Methods of modern mathematical physics. I: Functional analysis, 2nd ed., Academic Press [Harcourt Brace Jovanovich], New York, 1980. MR751959 (85e:46002)

[SW] L. Saloff-Coste and W. Woess, Transition operators, groups, norms, and spectral radii, Pacific J. Math. 180 (1997), no. 2, 333-367. MR.1487568 (99g:43005)

[W] W. Woess, Random walks on infinite graphs and groups, Cambridge Tracts in Math., vol. 138, Cambridge Univ. Press, Cambridge, 2000. MR1743100(2001k:60006)

Dipartimento di Matematica "G. Castelnuovo", Università di Roma "La Sapienza", Piazzale A. Moro 2, 00185 Roma, Italy

E-mail address: casadio@mat.uniroma1.it

Dipartimento di Matematica "G. Castelnuovo", Università di Roma "La Sapienza", Piazzale A. Moro 2, 00185 Roma, Italy

E-mail address: sandroft@mat.uniroma1.it 